\title{
THE SMALLEST MOUFANG LOOP REVISITED
}

\author{
PETR VOJTĚCHOVSKÝ
}

\begin{abstract}
We derive presentations for Moufang loops of type $M_{2 n}(G, 2)$, defined by Chein, with $G$ finite, two-generated. We then use $G=S_{3}$ to visualize the smallest non-associative Moufang loop.

Math. Subject Classification: Primary: 20N05, Secondary: 20F05

KEYWORDs: Moufang loop, presentations of loops, loops $M_{2 n}(G, 2)$.
\end{abstract}

\section{INTRODUCTION}

In order to derive a presentation for a groupoid $A=(A, \cdot)$, one usually needs to introduce a normal form for elements of $A$ written in terms of some generators. Such a normal form is not easy to find when $A$ is not commutative, and even more so when $A$ is not associative. Once a normal form is found, it might be still difficult to come up with presenting relations. Indeed, it is often the case that the only known presentation for a non-associative groupoid is the table presentation, i.e., the presentation consisting of all relations $x \cdot y=z$ such that $x \cdot y$ equals $z$ in $A$, and where $x, y$ run over all elements of $A$. Table presentations are extremely useful when one constructs a multiplication table for $A$, however, they are of little use when one needs to identify $A$ as a subgroupoid of another groupoid. To do the latter, it is necessary, in principle, to evaluate all products $x \cdot y$ with $x, y \in A$. It is therefore desirable to have access to presentations with a few presenting relations.

The infinite class of Moufang loops $M_{2 n}(G, 2)$, defined below, represents a significant portion of non-associative Moufang loops of small order. We derive compact presentations for $M_{2 n}(G, 2)$ for every finite, two-generated group $G$.

Thirty years ago, Chein and Pflugfelder [3] proved that the smallest non-associative Moufang loop is of order 12 and is unique up to isomorphism. It coincides with $M=M_{12}\left(S_{3}, 2\right)$. Guided by our presentation for $M$, we give a new, visual description of $M$ in the last section.

\section{The Loops $M_{2 n}(G, 2)$}

A loop $L=(L, \cdot)$ is Moufang if it satisfies one of the three equivalent Moufang identities

$$
x y \cdot z x=x(y z \cdot x), x(y \cdot x z)=(x y \cdot x) z, x(y \cdot z y)=(x y \cdot z) y .
$$

In fact, it is not necessary to assume that $L$ possesses a neutral element. By a result of Kunen [4], every quasigroup satisfying one of the Moufang identities is necessarily a (Moufang) loop. Every element $x$ of a Moufang loop has a two-sided inverse $x^{-1}$. Also, Moufang loops are diassociative, i.e, every two-generated subloop is a group. We will use these well-known properties of Moufang loops without warning throughout the paper.

The following construction is due to O. Chein [2]. Let $G$ be a finite group of order $n$. Pick a new element $u$, and define

$$
M_{2 n}(G, 2)=\left\{g u^{\alpha} ; g \in G, \alpha=0,1\right\},
$$

Work partially supported by Grant Agency of Charles University, grant number 269/2001/B-MAT/MFF. 
where

$$
g u^{\alpha} \cdot h u^{\beta}=\left(g^{(-1)^{\beta}} h^{(-1)^{\alpha+\beta}}\right)^{(-1)^{\beta}} u^{\alpha+\beta} \quad(g, h \in G, \alpha, \beta=0,1) .
$$

Then $M_{2 n}(G, 2)$ is a Moufang loop of order $2 n$. It is associative if and only if $G$ is commutative.

Let $\pi(m)$ be the number of isomorphism types of non-associative Moufang loops of order at most $m$, and let $\sigma(m)$ be the number of non-associative loops of the form $M_{2 n}(G, 2)$ of order at most $m$. Then, according to Chein's classification [2], $\pi(31)=13, \sigma(31)=8$, $\pi(63)=158, \sigma(63)=50$. (As Orin Chein kindly notified me, Edgar Goodaire noticed that the loop $M_{12}\left(S_{3}, 2\right) \times C_{3}$ is missing in [2]. He also observed that $M_{48}(5,5,5,3,3,0)$ is isomorphic to $M_{48}(5,5,5,3,6,0)$, and $M_{48}(5,5,5,3,3,6)$ to $M_{48}(5,5,5,3,6,6)$. That is why $\pi(63)$ equals 158 , rather than 159.) This demonstrates eloquently the abundance of loops of type $M_{2 n}(G, 2)$ among Moufang loops of small order.

\section{The Presentations}

We start with the table presentation (2) for $M_{2 n}(G, 2)$ and prove

Theorem 3.1. Let $G=\langle x, y ; R\rangle$ be a presentation for a finite group $G$, where $R$ is a set of relations in generators $x, y$. Then $M_{2 n}(G, 2)$ is presented by

$$
\left\langle x, y, u ; R, u^{2}=(x u)^{2}=(y u)^{2}=(x y \cdot u)^{2}=e\right\rangle,
$$

where $e$ is the neutral element of $G$.

Let us emphasize that (3) is a presentation in the variety of Moufang loops, not groups.

The complicated multiplication formula (2) merely describes the four cases

$$
\begin{aligned}
g \cdot h & =g h, \\
g u \cdot h & =g h^{-1} \cdot u, \\
g \cdot h u & =h g \cdot u, \\
g u \cdot h u & =h^{-1} g
\end{aligned}
$$

in a compact way. In particular, identities (7) and (5) imply

$$
u^{2}=e, g u=u g^{-1}(g \in G) \text {. }
$$

We claim that (8) is equivalent to (2). An element $g \in G$ will be called good if $g u=u g^{-1}$ can be derived from (3).

Lemma 3.2. If $h \in G$ is good, then (5) holds. If $g, h, h g \in G$ are good, then (6) holds. If $g, g^{-1} h$ are good, then (7) holds.

Proof. We have $g u \cdot h=(g u \cdot h) u \cdot u=(g \cdot u h u) u=\left(g \cdot h^{-1} u u\right) u=g h^{-1} \cdot u$ if $h$ is good. Assume that $g, h, h g$ are good. Then $g \cdot h u=g \cdot u h^{-1}=u \cdot u\left(g \cdot u h^{-1}\right)=u\left(u g u \cdot h^{-1}\right)=u \cdot g^{-1} h^{-1}=$ $h g \cdot u$. Finally, when $g$ and $g^{-1} h$ are good, we derive $g u \cdot h u=u g^{-1} \cdot h u=u \cdot g^{-1} h \cdot u=h^{-1} g$.

Thus (8) is equivalent to (2). Moreover, in order to prove Theorem 3.1, it suffices to show that every $g \in G$ is good.

Thanks to diassociativity, $g^{s}$ ( $s$ positive integer) is good whenever $g$ is. Since $G$ is finite, $g^{-1}$ is good whenever $g$ is.

Lemma 3.3. Assume that $g, h \in G$ are good. Then $g h$ is good if and only if $h g$ is. 
Proof. Because of the symmetry, it is enough to prove only one implication. Assume that $h g$ is good. By Lemma 3.2, $g \cdot h u=h g \cdot u$. Using this identity, we obtain $g h \cdot u g=g(h u \cdot g)=$ $(g \cdot h u) g=(h g \cdot u) g=h \cdot g u g=h u$, thus $g h=h u \cdot g^{-1} u=u h^{-1} \cdot g^{-1} u=u \cdot h^{-1} g^{-1} \cdot u$, and so $g h \cdot u=u \cdot h^{-1} g^{-1}$.

Lemma 3.4. Assume that $g, h \in G$ are good. Then so is $g h g$.

Proof. Since $g^{-1}, h$ are good, Lemma 3.2 yields $u g \cdot h=g^{-1} u \cdot h=g^{-1} h^{-1} \cdot u$. Then $u \cdot g h g \cdot u=(u g \cdot h) g \cdot u=\left(g^{-1} h^{-1} \cdot u\right) g \cdot u=g^{-1} h^{-1} \cdot u g u=g^{-1} h^{-1} g^{-1}$, and we are done.

We continue by induction on the complexity, or length, if you will, of the elements of $G$, defined below.

For $\varepsilon=1,-1$, let $X_{\varepsilon}$ be the set of symbols $\left\{x_{1}^{\varepsilon}, \cdots, x_{m}^{\varepsilon}\right\}$, and write $X=X_{1} \cup X_{-1}$. Every word $w$ of the free group $F=\langle X\rangle$ can be written uniquely in the form $x_{i_{1}}^{\varepsilon_{1}} \cdots x_{i_{r}}^{\varepsilon_{r}}$, where $i_{j} \neq i_{j+1}$, and $\varepsilon_{j}$ is a non-zero integer. Define the complexity of $w$ as the ordered pair $c(w)=\left(r, \sum_{j=1}^{r}\left|\varepsilon_{r}\right|\right)$, and order the complexities lexicographically.

From now on, assume that $G$ is two-generated, and write $x=x_{1}, y=x_{2}$.

Since $x u=u x^{-1}$ and $y u=u y^{-1}$ are presenting relations, both $x, y$ are good, and hence both $x^{s}, y^{s}$ are good for every integer $s$. The last presenting relation $x y \cdot u=u \cdot y^{-1} x^{-1}$ shows that both $x y$ and $y^{-1} x^{-1}=(x y)^{-1}$ are good. Then $y x$ and $x^{-1} y^{-1}=(y x)^{-1}$ are good, by Lemma 3.3. Also, Lemma 3.4 implies that $x^{-1} \cdot x y \cdot x^{-1}=y x^{-1}$ is good. Then $x^{-1} y$, $x y^{-1}=\left(y x^{-1}\right)^{-1}$ and $y^{-1} x=\left(x^{-1} y\right)^{-1}$ are good, by Lemma 3.3. This means that every $g \in G$ with $c(g)<(2,3)$ is good.

Lemma 3.5. Every $g \in G$ with $c(g)<(3,0)$ is good.

Proof. Suppose there is $g$ that is not good, and let $c(g)=(r, s)$ be as small as possible. We can assume that $g=a^{u} b^{v}$, where $\{a, b\}=\{x, y\}, s=|u|+|v|>2$, and $u \neq 0 \neq v$.

Either $|u|>1$ or $|v|>1$. Without loss of generality, $u>1$. (By Lemma 3.3, we can assume that $|u|>1$. When $u$ is negative, consider the inverse $b^{-v} a^{-u}$ instead, and apply Lemma 3.3 again.) Since $c\left(a^{u-2} b^{v}\right)<(2, s)$, the element $a^{u-2} b^{v}$ is good, and so is $a^{u-1} b^{v} a=a \cdot a^{u-2} b^{v} \cdot a$. As $a^{u-1} b^{v}$ is good by the induction hypothesis, $a^{u} b^{v} a=a \cdot a^{u-1} b^{v} \cdot a$ is good as well, by Lemma 3.4. Then the decomposition of $a^{u-1} b^{v} a$ into $a^{-1} \cdot a^{u} b^{v} a$ demonstrates that $a^{u} b^{v} a \cdot a^{-1}=a^{u} b^{v}$ is good, by Lemma 3.3. We have reached a contradiction.

To finish the proof, assume there is $g \in G$ that is not good, and let $c(g)=(r, s)$ be as small as possible. By Lemma 3.5, $r \geq 3$. When $r$ is odd, we can write $g=a^{\varepsilon_{1}} b^{\varepsilon_{2}} a^{\varepsilon_{3}} \cdots b^{\varepsilon_{r-1}} a^{\varepsilon_{r}}=$ $k h k$, where $k=a^{\varepsilon_{r}}, h=a^{\varepsilon_{1}-\varepsilon_{r}} b^{\varepsilon_{2}} a^{\varepsilon_{3}} \cdots b^{\varepsilon_{r-1}}$, and $\{a, b\}=\{x, y\}$. Since $c(k), c(h)<(r, s)$, both $k, h$ are good, and then $g$ is good by Lemma 3.4

Assume that $r$ is even. Then $g=a^{\varepsilon_{1}} b^{\varepsilon_{2}} \cdots a^{\varepsilon_{r-1}} b^{\varepsilon_{r}}=k h k$, where $k=a^{\varepsilon_{1}} b^{\varepsilon_{r}}$ and $h=$ $b^{\varepsilon_{2}-\varepsilon_{r}} a^{\varepsilon_{3}} \cdots b^{\varepsilon_{r-2}} a^{\varepsilon_{r-1}-\varepsilon_{1}}$. Again, $c(k), c(h)<(r, s)$, thus both $k$ and $h$ are good, and so is $g$, by Lemma 3.4.

Theorem 3.1 is proved.

\section{Visualization of the Smallest Moufang Loop}

The multiplication formula (2) for $M=M_{12}\left(S_{3}, 2\right)$ is certainly difficult to memorize, and so is the one in [5, Example IV.1.2]. We present a visual description of $M$.

Note that there are 9 involutions and 2 elements of order 3 in $M$ (cf. [1, Table 3]). We are going to define a 12-element groupoid $L$ and show that it is isomorphic to $M$. 
Look at the four diagrams in Figure 1. Think of the vertices $x_{0}, \ldots, x_{8}$ as involutions. Let $L$ consists of $e, x_{0}, \ldots, x_{8}, y, y^{-1}$, where $y$ is of order 3 . Interpret the edges of diagrams I-IV as multiplication rules in the following way. If $x_{i}$ and $x_{j}$ are connected by a solid line, let $x_{i} x_{j}$ be the third vertex of the (unique) triangle containing both $x_{i}$ and $x_{j}$. If $x_{i}$ and $x_{j}$ are not connected by a solid line, we must have $j=i \pm 3$, and then $x_{i}$ and $x_{j}$ are connected by a dotted line (in diagram III). Define $x_{i} x_{i+3}=y$ and $x_{i} x_{i-3}=y^{-1}$.
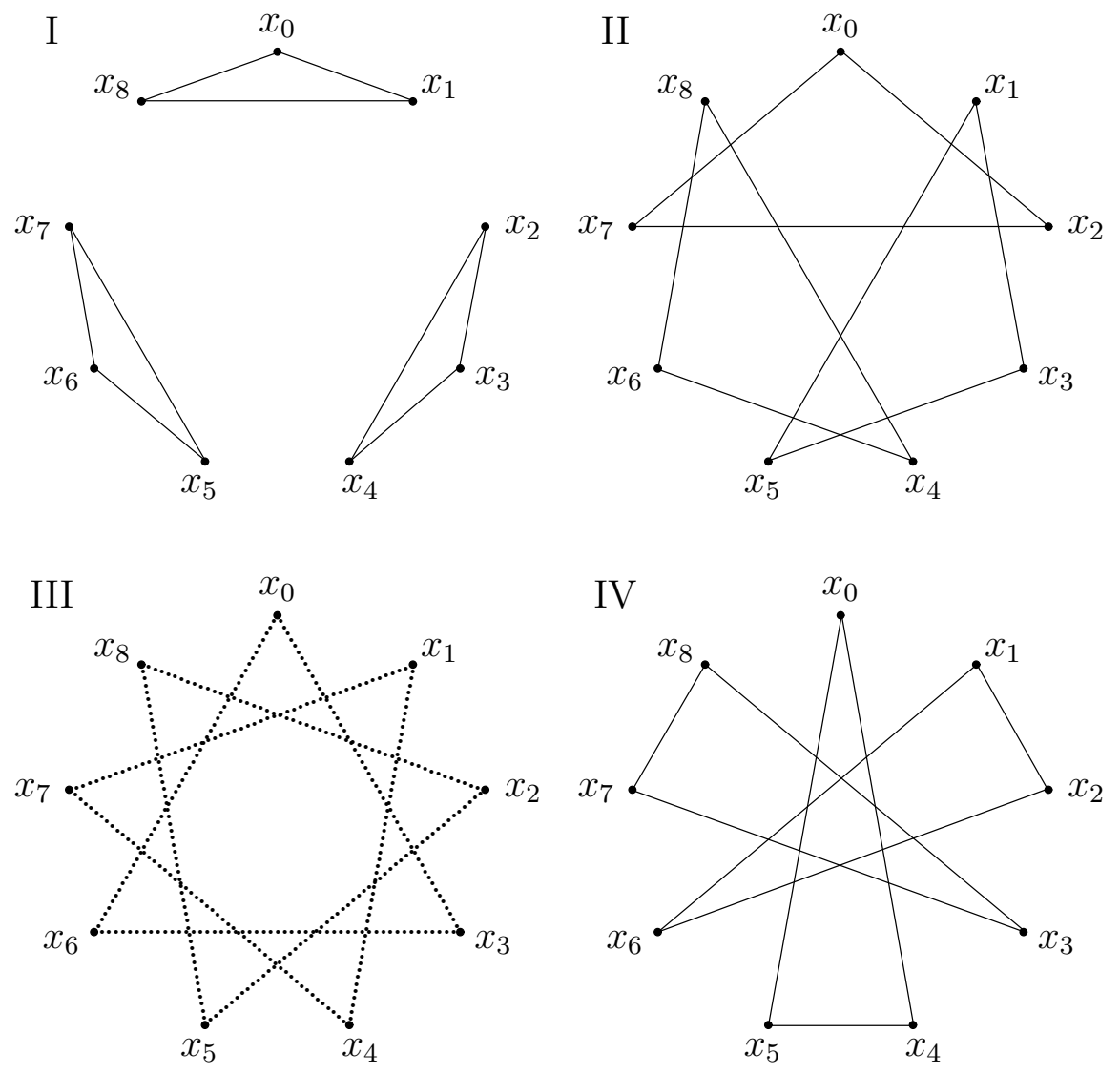

Figure 1. Multiplication in $M_{12}\left(S_{3}, 2\right)$

This partial multiplication can be extended by properties of Moufang loops. To avoid ambiguity, we postulate that $x_{i} y=y^{-1} x_{i}=x_{i+3}$ and $y x_{i}=x_{i} y^{-1}=x_{i-3}$.

Obviously, $L$ is closed under multiplication and has a neutral element. It is non-associative, since $x_{0} x_{1} \cdot x_{3}=x_{8} x_{3}=x_{7} \neq x_{4}=x_{0} x_{5}=x_{0} \cdot x_{1} x_{3}$. Is $L$ isomorphic to $M$ ? There is a unique Moufang loop of order 12 [3], so it suffices to check the Moufang identities for $L$. However, this is not so easy! Instead, we verify directly that $L$ satisfies the multiplication formula (2) with some choice of $G$ and $u$.

Remark 4.1. It does not suffice to verify (8) for some choice of $G$ and $u$ because (8) is equivalent to (2) only when it is assumed that $L$ is Moufang.

Put $x=x_{0}$, and observe that $G=\langle x, y\rangle=\left\{e, x_{0}, y, x_{3}, x_{6}, y^{-1}\right\}$ is isomorphic to $S_{3}$. Let $u=x_{1} \notin G$. We show that (44)-(7) are satisfied for every $g, h \in G$. Thanks to the symmetry of Figure 1, it is enough to consider only $\{g, h\}=\left\{x_{0}, x_{3}\right\},\left\{x_{0}, y\right\}$. 
Identity (4) is trivial. Let us prove (5). We have $x_{0} x_{1} \cdot x_{3}=x_{8} x_{3}=x_{7}=y x_{1}=x_{0} x_{3}^{-1} \cdot x_{1}$, $x_{0} x_{1} \cdot y=x_{8} y=x_{2}=x_{6} x_{1}=x_{0} y^{-1} \cdot x_{1}, x_{3} x_{1} \cdot x_{0}=x_{5} x_{0}=x_{4}=y^{-1} x_{1}=x_{3} x_{0}^{-1} \cdot x_{1}$, and $y x_{1} \cdot x_{0}=x_{7} x_{0}=x_{2}=x_{6} x_{1}=y x_{0}^{-1} \cdot x_{1}$. Similarly for (6), (17).

Hence $L$ is isomorphic to $M$. The subloop structure of $L$ is apparent from the visual rules, too. If $j \equiv i(\bmod 3)$ then $\left\langle x_{i}, x_{j}\right\rangle \cong S_{3}$; otherwise, $\left\langle x_{i}, x_{j}\right\rangle \cong V_{4}$, for $i \neq j$.

\section{REFERENCES}

[1] O. Chein, Moufang Loops of Small Order I, Trans. Amer. Math. Soc. 188 (1974), 31-51.

[2] O. Chein, Moufang Loops of Small Order, Memoirs of the American Mathematical Society, Volume 13, Issue 1, Number 197 (1978).

[3] O. Chein, H. O. Pflugfelder, The smallest Moufang loop, Arch. Math. 22 (1971), 573-576.

[4] K. Kunen, Moufang Quasigroups, J. Algebra 183 (1996), no 1, 231-234.

[5] H. O. Pflugfelder, Quasigroups and Loops: Introduction, (Sigma series in pure mathematics; 7), Heldermann Verlag Berlin (1990).

Department of Mathematics, University of Denver, 2360 S Gaylord St, Denver, CO 80208, USA

E-mail address: petr@math.du.edu

Eingegangen am 13. März 2003 